\title{
Incidence rate of breast cancer, clinical and ultrasound approaches to diagnosing the same in dogs
}

\author{
V. Salautin, V. Gorinsky, A. Molchanov, G. Demkin, N. Pudovkin* and S. Salautina \\ Department of Animal Morphology, Pathology and Biology, Faculty of Veterinary Medicine, Saratov State Agrarian \\ University named after N.I. Vavilov, Russia, Saratov, 410012, Teatralnaya square, 1, ${ }^{*}$ Email: niko-pudovkin@yandex.ru
}

(Received December 21, 2017; Accepted April 20, 2018)

\begin{abstract}
Mammary gland tumors in dogs rank second among all newly diagnosed tumors in this species. Research goal was to study clinical/morphological characteristics of breast cancer in dogs with the use of ultrasound investigation. 34 dogs of different breeds and age groups with spontaneously emerging neoplasms of mammary glands were chosen for the study. To classify malignant tumors, the histological classification was applied. Complete examination of breast tumors was carried out with the help of clinical observation, ultrasound, radiologic, hematological and ultrasonographic methods applied. The largest percentage $(23.5 \%)$ of animals with mammary gland neoplasms are mongrel female dogs. The breed predisposition was detected in dogs of the following breeds: Miniature Schnauzer - 11.9\%, Standard Dachshund - 11.9\%, American Bulldog, American Cocker Spaniel and Miniature Poodle - 8\% each. Breast cancer was diagnosed in 29 animals (85.3\%), 100\% of those cases in the nodular form with microcalcifications in the tumor parenchyma. The horizontal orientation of growth is more often typical of breast cancer in the mammary gland pairs I and II at the initial stage of carcinogenesis, and is characterized by an inhomogeneous hypoechoic structure with sufficiently pronounced boundaries detected during the ultrasound investigation. The vertical orientation of growth is mainly typical of breast cancer in the mammary gland pairs III, IV and V from the very beginning of the disease. The ultrasonography usually shows: inhomogeneous hypoechoic structure containing inclusions in the form of cords or foci of a higher acoustic solidity; also clearly defined lateral acoustic shadows and dorsal echo enhancement; as well as unevenness and indistinctness of borders. The performed histological study within the current research revealed a multifocal, infiltrating epithelial neoplasm with distinct edges, surrounded by a large amount of fibrous tissue. The tumor consisted of a number of acini with a tendency of forming a network structure. The shape of cells was detected from round to irregular, the cytoplasm was found scarce, often with uneven borders, with the large central core, with chromophilic substance and 1-2 nucleoli. The cells were characterized by the pronounced anisocytosis and anisocariosis, and a great number of mitotic figures. Lymphatic invasion was observed. Ultrasound investigation of the mammary gland allows for performing percutaneous minimally invasive interventions and differential diagnostics of cystic and solid lesions of the mammary gland (96-100\% accuracy), mastopathy and carcinoma conditions.
\end{abstract}

Keywords: Prevalence, Dogs, Cancerts, Diagnosis

Available online at http://www.vetmedmosul.org/ijvs

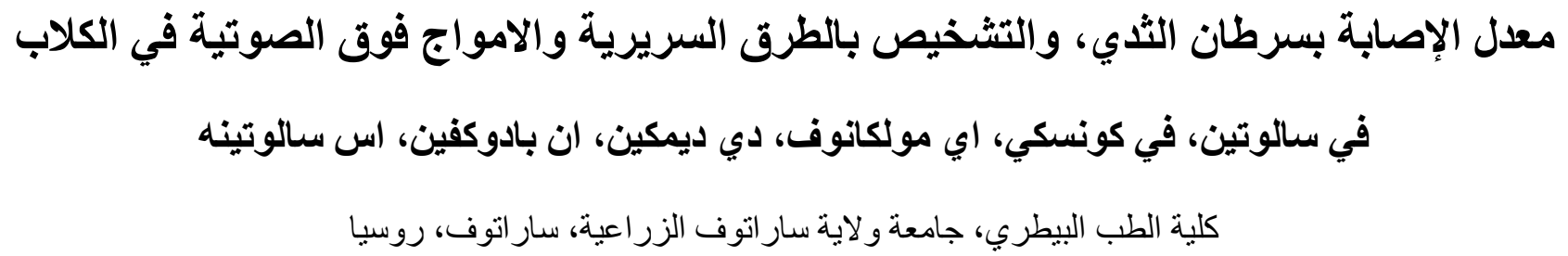

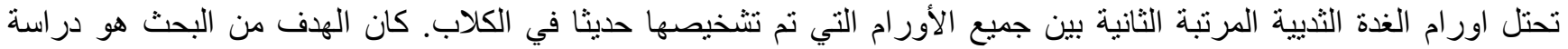

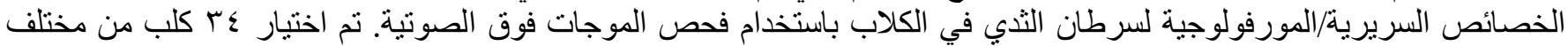




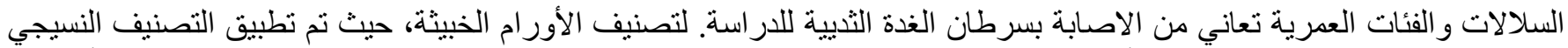

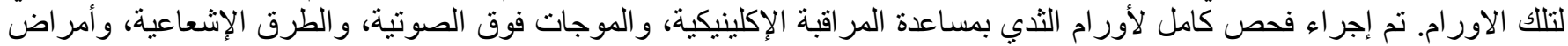

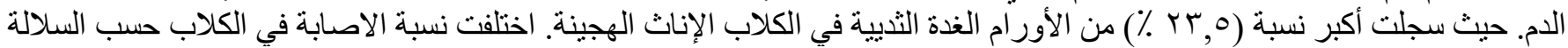

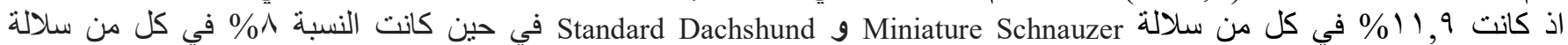

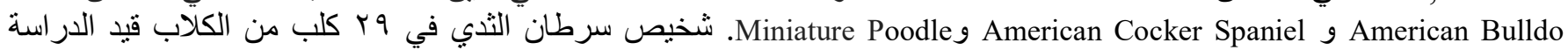

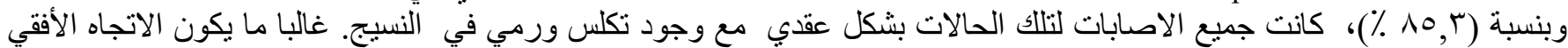

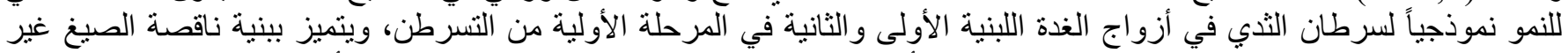

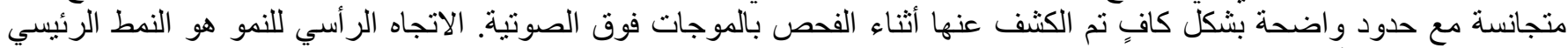

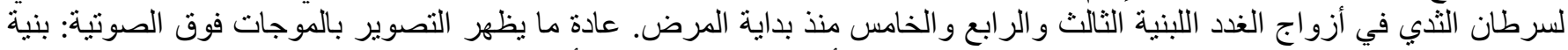

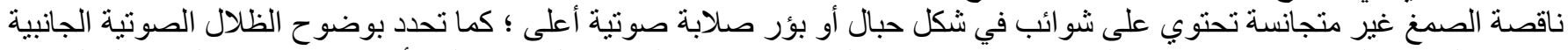

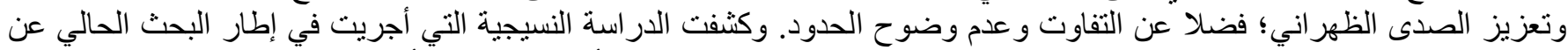

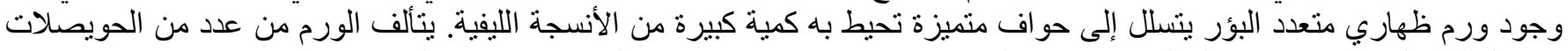

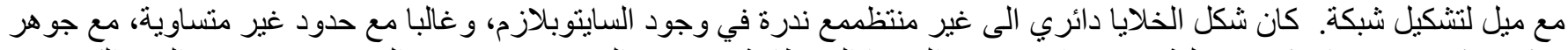

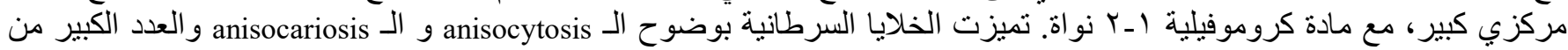

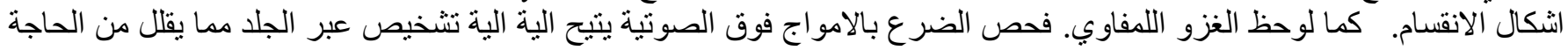

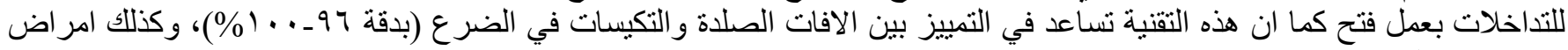

$$
\text { وسرطانات الضرع. }
$$

\section{Introduction}

Mammary gland tumors (MGT) in dogs have a rather high incidence rate and rank second among all newly diagnosed tumors in this species. Based on published literature, the risk of developing breast cancer (BC) in dogs is at least $50 \%$ of all cases of mammary gland (MG) neoplasms (1). The risk group is performed mainly by unneutered female dogs at the age from 5 to 16 years, and the peak incidence comes on animals from their middle age up to 10 years $(2,3)$.

Epithelial malignant tumors in dogs (up to $90 \%$ ) are characterized by the structure heterogeneity $(4,5)$. BC prognosis depends on many factors, including the age of the animal, the localization and features of tumor growth, its histological type, the presence of metastases, etc.

Research goal was to study clinical and morphological characteristics of breast cancer in dogs with the use of ultrasound investigation.

\section{Materials and methods}

Clinical observations and studies were conducted from 2014 to 2017 at the premises of the pet clinic at the Pet Center Zoostil (Volgograd, Russia) and the Department of Morphology, Animal Pathology and Biology of the Saratov State Agrarian University.

Dogs of different breeds, sex and age groups with spontaneously emerging neoplasms of mammary glands were chosen for the study. Upon admission to the clinic, animals with any visual signs of breast neoplasms were selected for further studies and observations. No animals died during the research period.

To confirm the diagnosis, cytological studies using the method of fine needle aspiration biopsy (FNAB) were performed at primary admission. When using surgery as a treatment method, a morphological examination in the form of a histological study of tissue samples was performed in $100 \%$ of cases.

Ultrasound investigation was performed with the use of the ultrasonic medical diagnostic device 'Ultrasonic Diagnostic System SonoScape A-6 Vet.' and with the help of transducers 'micro-curvedarray C612' and 'lineararrayL745'. To examine superficial neoplasms, a high-frequency transducer 'lineararrayL745' (center frequency $7.5 \mathrm{MHz}$, frequency range $10-5 \mathrm{MHz}$, pitch $0.360 \mathrm{~mm}$, angle/width $46 \mathrm{~mm}$ ) was used. To determine the nature of the blood flow, Doppler ultrasonography was performed with the use of 'ESAOTE MyLab40Vet' scanner and a 4-7.5 $\mathrm{MHz}$ multi-frequency phased transducer. Neoplasms were examined three-dimensionally, which made it possible to more accurately determine the size of neoplasms and their echostructure features.

Radiographic examination was carried out with the use of the mobile X-ray ward apparatus 'Arman 10Л6-011' and 'ORANGE-1040HF EcoRay'. The X-ray examination produced images of two types: the right lateral view (with thoracic extremities positioned headward) and dorsoventral view. When analyzing X-ray images, the attention was drawn to the anatomical and topographical features of the contours of the heart, lungs, respiratory tracts and large blood vessels. 
Hematologic studies were carried out with the use of the hematological analyzer 'Vet Auto Hematology Analyzer Mindray BC-2800', while biochemical blood tests were carried out with the use of the semi-automatic analyzer 'BioChem SA'.

\section{Research findings}

During the research period 3240 dogs with diseases of various etiologies were clinically examined, among them $185(5.7 \%)$ were diagnosed with oncology diseases. In $100 \%$ of cases $(n=34)$, mammary gland tumors were recorded in female dogs, among them the number of sterilized animals was $5.9 \%(\mathrm{n}=2)$.

Based on our research findings, female dogs of the following age groups were found prone to the development of MGT: up to 8 years $-14.7 \%(\mathrm{n}=5)$, from 8 to 12 years $73.6 \%(\mathrm{n}=25)$, from 12 to 16 years $-8.8 \%(\mathrm{n}=3)$, and over 16 years $-2.9 \%(\mathrm{n}=1)$.

After studying the issue of breed predisposition to $\mathrm{MG}$ neoplasms in female dogs, we obtained the following results, presented in Table 1.

Table 1: Breed predisposition to mammary gland neoplasms $(\mathrm{n}=34)$

\begin{tabular}{lcc}
\hline Breed & $\begin{array}{c}\text { Number of } \\
\text { animals }\end{array}$ & $\begin{array}{c}\text { Rate } \\
(\%)\end{array}$ \\
\hline Miniature Schnauzers & 4 & $11(9)$ \\
Standard Dachshunds & 4 & $11(9)$ \\
American Bulldogs & 3 & $8(8)$ \\
American Cocker Spaniels & 3 & $8(8)$ \\
Miniature Poodles & 3 & $8(8)$ \\
Yorkshire Terriers & 2 & $5(9)$ \\
German Shepherd & 2 & $5(9)$ \\
mixed breed dogs & 1 & $2(9)$ \\
Pugs & 1 & $2(9)$ \\
Russian Toy Terriers & 1 & $2(9)$ \\
Jagdterriers & 1 & $2(9)$ \\
Dobermanns & 1 & $2(9)$ \\
Airedale Terriers & 8 & $23(5)$ \\
Mongrel dogs & 34 & 100 \\
\hline Total & & \\
\hline
\end{tabular}

Based on the data in Table 1, mongrel female dogs constitute the largest percentage $(23.5 \%)$ of animals with MG neoplasms. Besides, the obtained results evidence the following breed predisposition to MG neoplasms in dogs: the Miniature Schnauzer makes $11.9 \%$, the Standard Dachshund makes $11.9 \%$, the American bulldog, the American Cocker Spaniel and the Miniature Poodle make $8 \%$ each.

Based on the results of morphological studies performed, benign tumors of mammary glands were revealed in $14.7 \%(\mathrm{n}=5)$ cases. At the same time, breast cancer was diagnosed in $85.3 \%(\mathrm{n}=29)$ animals.

Based on the results of histological studies of tissue samples gathered during surgery $(\mathrm{n}=27)$, the following neoplasms were detected depending on their morphological type: adenocarcinoma in $74.1 \%$ of cases $(n=20)$, solid carcinoma in $4.8 \%$ of cases $(n=4)$, sarcoma in $7.4 \%$ of cases $(\mathrm{n}=2)$ and mammary carcinosarcoma in $3.7 \%$ of cases $(\mathrm{n}=1)$.

The performed histological study of the tissue samples showed that adenocarcinoma was the prevailing type of the detected neoplasms. As per microscope field of view, a multifocal infiltrating epithelial neoplasm with distinct edges, surrounded by a large amount of fibrous tissue was detected. The tumor consisted of a number of acini with a tendency of forming a network structure. The acini were lined with 1-3 layers of cells and had a thin vascular fibrous stroma. The shape of cells was detected from round to irregular, the cytoplasm was scarce, often with uneven borders, with the large central core, with chromophilic substance and 1-2 nucleoli. The cells were characterized by the pronounced anisocytosis and anisocariosis, and a great number of mitotic figures (up to 3-5 per high power field). Lymphatic invasion was observed.

According to histopathological differentiation, the diagnosed tumors were distributed as follows: $22.2 \%$ of cases $(n=6)$ with a high degree of differentiation, $40.8 \%$ of cases $(n=11)$ with an average degree of differentiation, and $37 \%$ of cases $(n=10)$ with a low degree of differentiation.

According to histopathological differentiation, tumors with a moderate degree of differentiation were the most frequent ones. They were detected with lesions of extensive growth of abnormal tumor tissue, those lesions being separated from each other by thin connective tissue layers. Tumor tissue consisted of small cells with scanty cytoplasm, large nuclei, abundant chromatin condensation, mitotic figures. There were also found multinucleated cells, focal necrosis, focal hemorrhages in the tumor tissue. Stroma in most cases was moderately pronounced. Moderate blood vessel filling, stasis and erythrocyte sludge was registered. Single atrophied glands with a preserved histostructure were detected in tumor and perilesional tissue within a loose stroma.

Metastatic lesions of regional lymph nodes were detected in $17.2 \%$ of cases $(\mathrm{n}=5)$ at the primary admission. Remote metastases were not diagnosed either by X-ray or ultrasound investigation.

During the research period, 29 female dogs were diagnosed a nodular form of BC, constituting $85.3 \%$ cases of all MGT and $100 \%$ cases of all animals diagnosed with BC. Tumors burdened with hematoma formation were detected in $10.3 \%$ of cases $(n=3)$, (Figures 1 and 2 ).

Figure 2 clearly displays the hypoechoic, heterogeneous structure of the tumor parenchyma with hyperechoic 
microcalcinations providing an acoustic shadow. The neoplasm is surrounded with a cavity with anehogenous contents (blood). Dorsal echo enhancement is also clearly displayed.

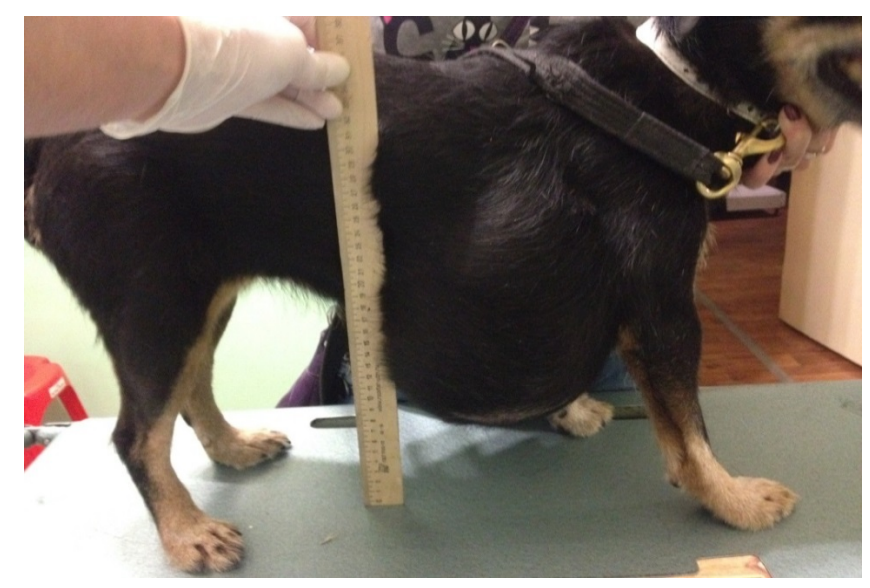

Figure 1: Adenocarcinoma of the mammary gland burdened with the hematoma formation. Jagdterrier female dog of 12 years old.

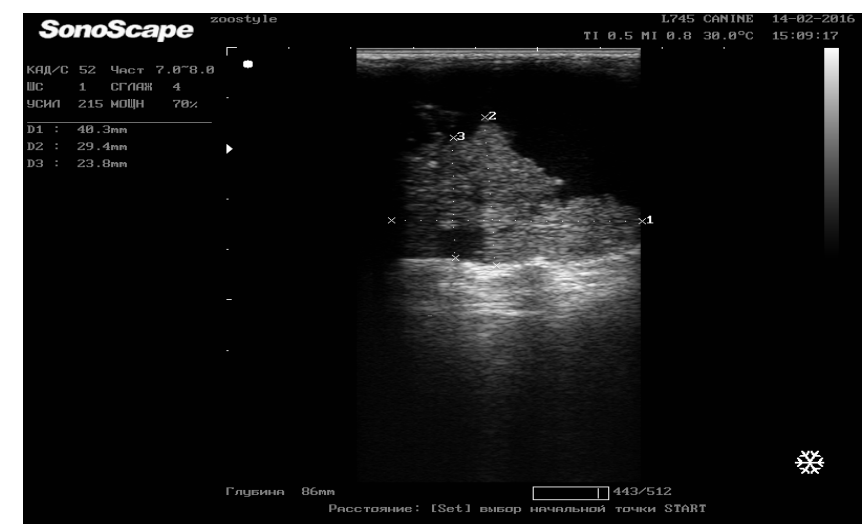

Figure 2: Ultrasound image of adenocarcinoma of the mammary gland burdened with the hematoma formation. Jagdterrier female dog of 12 years old.

In terms of number of tumor nodes in MG per one animal, the following pattern was revealed: one tumor node per animal in $58.6 \%$ of cases $(n=17)$ and more than one node per animal in $41.4 \%$ of cases $(n=12)$.

The prevalence rate of tumor nodes was also determined depending on their location in MG pairs: in pairs I, II - in $31 \%$ of cases $(n=9)$ and in pairs III, IV, V - in $69 \%$ of cases $(\mathrm{n}=20)$.

The horizontal orientation of growth is more often typical of breast cancer in the mammary gland pairs I and II in the initial stage of carcinogenesis, and is characterized by an inhomogeneous hypoechoic structure with sufficiently pronounced boundaries detected during the ultrasound investigation (Fig. 3).

The vertical orientation of growth is mainly typical of breast cancer in the mammary gland pairs III, IV and V from the very beginning of the disease. The ultrasonography shows inhomogeneous hypoechoic structure containing inclusions in the form of cords or foci of a higher acoustic solidity; also clearly defined lateral acoustic shadows and dorsal echo enhancement; as well as unevenness and indistinctness of borders (Fig. 4).

The presence of microcalcifications in the tumor parenchyma is rather characteristic of the nodular form of $\mathrm{BC}$ in dogs (Fig. 5).

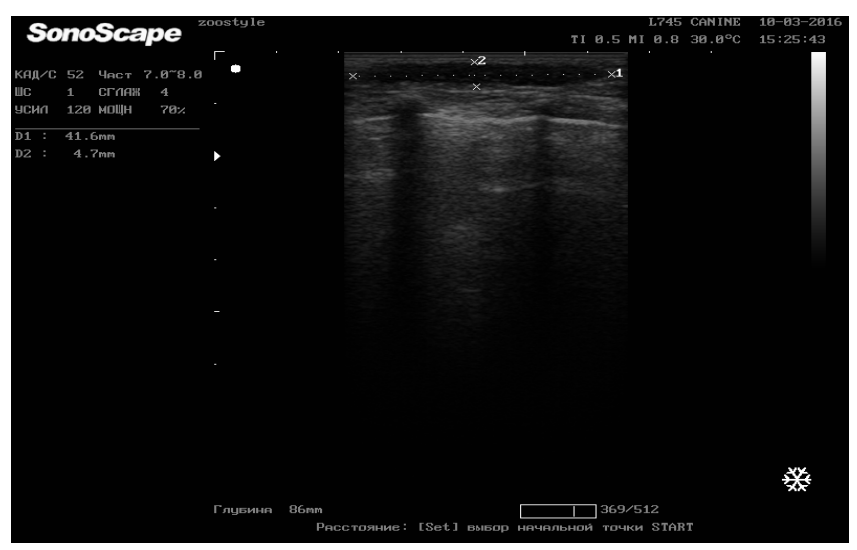

Figure 3: Ultrasound image of adenocarcinoma localized in the mammary gland pair I. Miniature Schnauzer female dog of 9 years old.

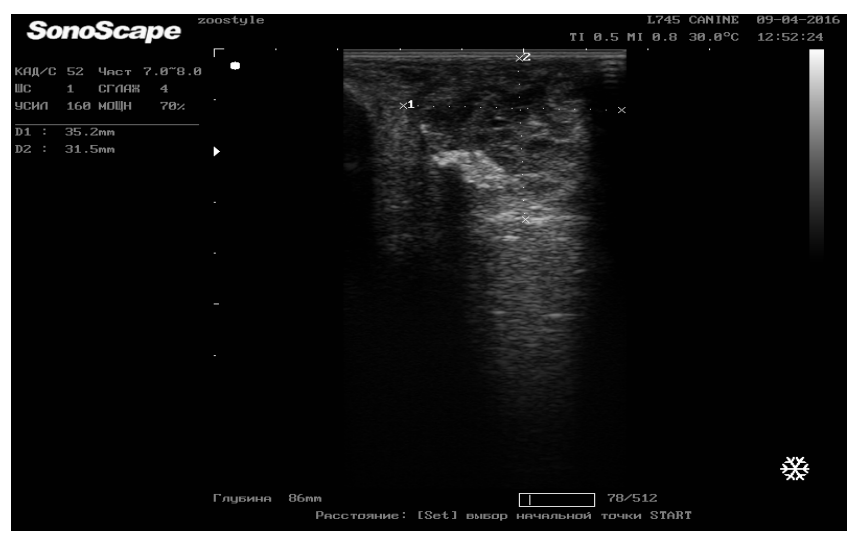

Figure 4: Ultrasound image of a nodular form of adenocarcinoma localized in the mammary gland pair IV. Standard Dachshund female dog of 10 years old.

There are four types of vascular pattern based on the nature of vascularization. In case of the third type, both peri- and intranodular (mixed) blood flow is detected; while the fourth type is characterized by intranodular blood flow 
only. The presence of the $3^{\text {rd }}$ and $4^{\text {th }}$ types of the tumor vascularization is generally regarded as an indirect sign of a malignant process.

While examining 29 female dogs with a morphologically confirmed diagnosis of $\mathrm{BC}$, the intra- and perinodular blood flow was recorded in 26 animals $(89.7 \%)$, while perinodular blood flow was recorded in 3 dogs $(10.3 \%)$. Thus, the intra- and perinodular (mixed) type of blood flow is evidenced as the most characteristic one of $\mathrm{BC}$ in female dogs (Fig. 6).

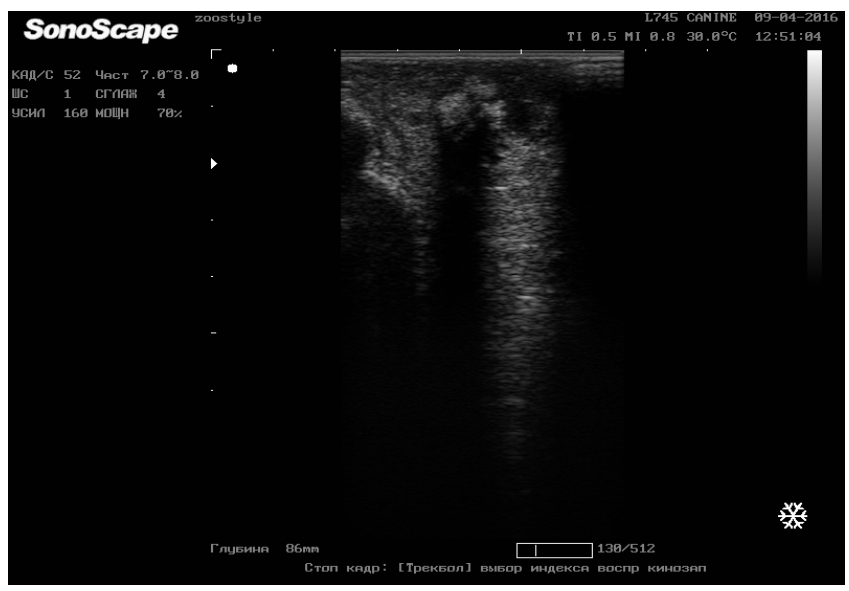

Figure 5: Ultrasound image of microcalcifications in the adenocarcinoma parenchyma of MG. German Shepherd mixed breed female dog of 9 years old.

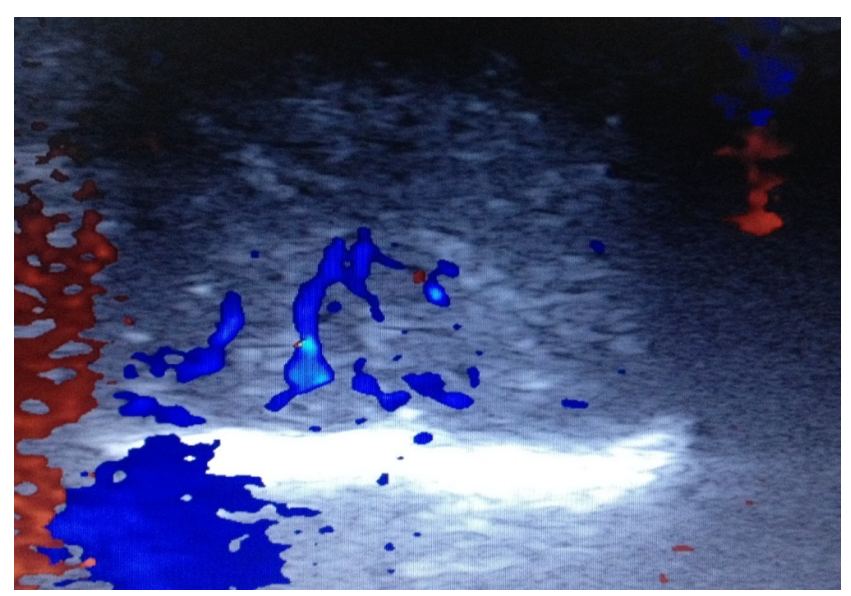

Figure 6: Doppler ultrasonography. III stage breast cancer. Example of the intra- and perinodular (mixed) type of blood flow (type 3).

\section{American Bulldog female dog of the age 9 years 7 months}

The results obtained through the hematological examination show an increase in the platelets number up to
$662.56 \pm 37.9 \times 10^{9} / \mathrm{L}$ in dogs diagnosed with stage II and stage $\mathrm{BC}$ as compared to normal $458.99 \pm 28.4 \times 10^{9} / \mathrm{L}(\mathrm{p} \leq$ 0.05 ). No statistically significant differences in the number of erythrocytes and leukocytes were detected.

The results obtained through the biochemical blood test show an increase in the total protein by $12 \%(80.6 \pm 3.02$ $\mathrm{g} / \mathrm{l})$ as compared to normal $71.87 \pm 4.89 \mathrm{~g} / \mathrm{l},(\mathrm{p} \leq 0.05)$. Such blood values as glucose, SGOT (serum glutamic oxaloacetic transaminase), SGPT (serum glutamic pyruvic transaminase), total bilirubin, creatinine, urea, calcium and phosphorus did not show any statistically significant differences as compared to the normal values.

\section{Discussions}

MG neoplasms are quite widespread and rank third after lymphomas and skin tumors (6).

Neoplastic diseases in dogs are quite widely spread within the examined territory. A high percentage of diagnosed neoplasms in animals is apparently caused by emissions from the metallurgical, chemical and fuel industry enterprises located within the urban territory, as well as by an increased content of nitrogen oxides, formaldehyde and phenol in the air.

Ultrasound investigation as a diagnostic method is constantly increasing its potential due to the improvement of existing techniques and the development of new ones. Ultrasonic mammography as one of the methods for diagnosing MG neoplasms has shown its high efficacy (6). The most essential signs of a nodular form of $\mathrm{BC}$ at the ultrasound investigation are as follows: an irregular shape, mainly anechoic structure, unclear boundaries, dorsal echoic enhancement, and lateral acoustic shadows. Currently an ultrasound investigation enables to diagnose cystic and solid lesions of MG (96-100\% accuracy rate), mastopathy and carcinoma. This kind of investigation also makes it possible to perform minimally invasive percutaneous interventions under the visual control.

\section{References}

1. Argyle DJ, Brearley MJ, Turek MM. Decision Making in Small Animal Oncology B.:Wiley-Blackwell. 2008;pp:408.

2. Moore AS, Frimberger AE. Oncology for veterinary technicians and nurses. A John Wiley \& Sons, Inc. 2010;pp:264-272.

3. Morris J, Dobson J. Small Animal Oncology. British: Blackwell Science Ltd, 2001;p.1-3.

4. Bomhard von D. Praxis der Onkologie bei Hund und Katze Stuttgard: In Nolte I \& Nolte Epidemiologie. Enke; 2001;pp:23.

5. Donatas S, Pockevičius A, Mačiulskis P, Šimkienè V, ZorgevicaPockeviča L. Pathomorphological analysis of the most common canine skin and mammary tumours. National Food Vet Risk Assessm Institute. 2015;pp: 63-70.

6. Withrow SJ, Vail DM, Page RL. Small Animal Clinical Oncology 5th Edition. Philadelphia: W. B. Saunders Company; 2013;pp.750. 